\title{
Article \\ Reflection Symmetry Axes Localization Framework for Intelligent Object Perception Based on Salient Symmetry Feature
}

\author{
Kaichao Wu ${ }^{1,+} \mathbb{D}$, Yuan $Q i^{1,+}$, Yunju Ma ${ }^{1,+}$,Cheng Liu ${ }^{1,+}$ and Dazhi Jiang ${ }^{1,+* \mathbb{D}}$ \\ 1 Department of Computer Science, Shantou University, China 515063. \\ 2 Intelligent Manufacturing Key Laboratory of Ministry of Education, Shantou University, China 515063. \\ * Correspondence: dzjiang@stu.edu.cn
}

\begin{abstract}
Computationally detecting the reflection symmetry axis of a given images has been actively studied recently because of its wide applications in subfields of computer vision such as object detection, object perception, and feature engineering. In this paper, we present an optimized feature-centered reflection symmetry axis detection framework for symmetry object perception. The proposed framework starts with a salient symmetry feature which is particularly designed for fitting a specific symmetry property, that is, the reflection symmetry object is usually prominent in a wild scene, catching human's eyes. After optimizing the candidate feature pairs with feature descriptor, a Symmetric Transformation Matrix (STM) is computed based on a group of optimal salient symmetry feature matching pairs, which can be explicitly viewed as an abstract representation of the constraint regions of symmetry objects in an image. By analyzing the STM rank, a set of potential symmetry axis is efficiently detected. Two experiments have been investigated on different datasets, which show that the performance of the existing algorithms embedded with the proposed salient symmetry feature have improvements and the detecting framework can be competitive compare with state-of-the-art method, respectively.
\end{abstract}

Keywords: Object perception; Reflection symmetry; Feature matching

\section{Introduction}

Reflection symmetry is a manifestation of an object that two parts of which mirror each other. Among objects in wild, reflection symmetry is one of the pervasive symmetry types (Four types of symmetry in two-dimensional Euclidean space: reflection symmetry ${ }^{1}$, rotational symmetry ${ }^{2}$, translational symmetry ${ }^{3}$, and glide-reflection symmetry ${ }^{4}$ ). Reflection symmetry of symmetry object also makes the object easy to recognize and helps some objects look more attractive.

Since objects with reflection symmetry are ubiquitous, researches on reflection symmetry has become active in the field of computer vision. Apart from the various applications into high-level tasks such as face recognition [1], human perception[2] ,texture synthesis [3], 3D modeling [4] ,robotic vision [5]and sensor-based motion recognition[6],reflection symmetry as a kind of balance and harmony feature, comparing to other lower geometric features like color and line, has been extensively studied into shape analysis[7-9], local and global features[10-13], image segmentation [14,15],salient detection[16,17] and affective computing [18].

Intrigued by the balance symmetry feature [18] which is abstracted from the reflection symmetry acting as a high-level feature, we expected to detect the reflection symmetry axis of an image which

\footnotetext{
https://encyclopedia.thefreedictionary.com/reflection+symmetry, if without special indication, the symmetry refers to reflection symmetry for short in this paper

2 https://encyclopedia.thefreedictionary.com/rotational+symmetry

https://encyclopedia.thefreedictionary.com/translational+symmetry

https://encyclopedia.thefreedictionary.com/Glide+reflection
} 
exhibits symmetry objects. In general, reflected symmetry axis detection algorithms can be roughly divided into two categories [10]: parameter-centered[10,19,20] and feature-centered [10,12,21-28]. The feature-centered methods, as the name suggested, is based on an image feature (or several features, no matter what kind of features), it calculates the feature firstly, and then estimates the required parameters of the symmetric pattern. A rich body of work for detecting reflection symmetry axis falls in feature-centered. For example, Loy and Eklundh [12] use the scale invariant feature transform (SIFT) feature to map mirror SIFT feature and then detect the reflection symmetry axis based on the Hough voting scheme [29]. Kondra S et al. [25] proposed an algorithm based on multi-scale operator, it calculates the correlation measure (concludes SIFT related method) along the discrete direction, searching a match on the direction and identifying the region for marches in scale space. Patraucean $\mathrm{V}$ et al. [26] used a kind of edge descriptors to detect symmetry patterns and in [27], a combinational technology of Gesalt algebra based on SIFT descriptors was proposed. While most of these algorithms focus on feature matching pairs searching [28] or symmetric parameter optimizing [30], which means that there needs a large number of alternative matching feature to maximize the solvable domain. SIFT [31] feature-centered algorithms [12,21,25-28,30] offer a considerable solution. However, although a large collection of interesting points (like SIFT points) guarantees that the details of image can be persevered to the highest degree for detecting symmetry axis accurately, the fact is that only one pair of geometrically symmetric points is sufficient to determine the symmetry axis if minimizing the presence of noise, illumination variations, etc. Also, the feature themselves are irrelevant. There is a misconception that any other image features can be applied to detect symmetry axis, such as SURF (Speeded Up Robust Features) [20], PAC-SIFT [32] (Even though nobody has done this so far). There also exist other optimized SIFT features algorithms like [21]. These kinds of features might do enjoy high performance predictably, but the importance and particularity of the feature are not taken into account, and the illustration why the feature is selected or what the beneficial effects of these features are is lacked. As a result, the "semantic gap" between the lower level and the higher visual tasks is ignored.

These considerations above motivate us to develop an informative feature with strong generalization, named salient symmetry feature in this paper, targeting for symmetry detection. The motivation of the method comes from that humans pay more attention to salient symmetry objects when interpreting a symmetry-contained scene (i.e.humans are sensitive to the symmetry) [11]. An image is a mapping of a complex real scene in two-dimension space, humans can quickly recognize the reflection symmetry structures of an image which exhibits symmetry objects, especially the image contains multiple symmetry properties [33], and it has been proved that human's eyes fixations concentrate more on the symmetry axis [16]. Stemmed from these facts, this paper develops a new feature extraction method based on saliency detection, and incorporates it with a fitting matrix to optimize the reflection symmetry axis detection.

Basically, the feature extraction method is inspired from an improved Multi-scale symmetry model - a kind of visual saliency model[16] which tries to find symmetry pattern in eyes fixation by applying isotropic symmetry operator[13] on each pixels of an image. Specifically, the core idea of this model, which uses the isotropic symmetry operator to calculate the potential symmetry pattern, is retained, but it is implemented on our initial salient points, i.e., the extrema of local area at each scale in scale-space, rather than all pixels. The local area which the salient point is in refers to symmetry kernel. Different from the work using the isotropic symmetry operator as a detector, this paper view it as a filter. By assigning symmetry magnitude and symmetry orientation to each candidate through isotropic symmetry operator, these salient points which highly response to local symmetry pattern are reserved as final feature point and named as salient symmetry point, the noise of none-feature point are filtered accordingly. The purpose of this step is to activate the pixels which have positive effect for reflection symmetry axis detection in different scales, but avoid high complexity and in the meantime, enhance the pertinence and adaption of extracted pixels. Since the size of symmetry kernel can limit the degree of the symmetry pattern the feature point response to, this paper also develops a region 


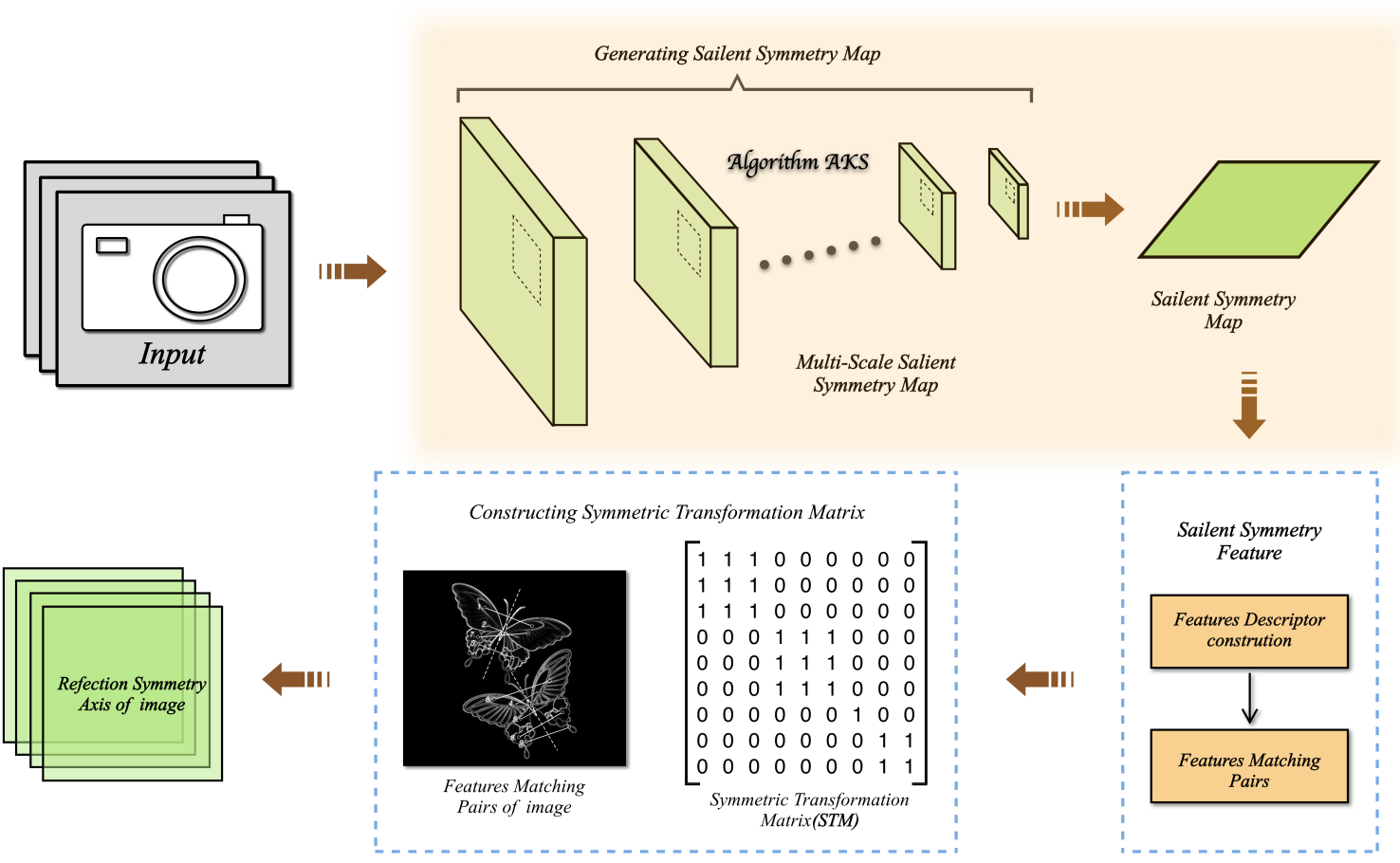

Figure 1. Pipeline of the complete optimization framework for the detection of reflection symmetry axes. Given an input image, salient symmetry map which comprised of salient symmetry points is generated, and a feature descriptor is constructed to describe feature points for feature matching pairs. Then a unique symmetric transformation matrix is calculated for detecting reasonable refection symmetry axis.

growing method to adjust it adaptively. Based on the extracted salient symmetry point, the exploited descriptor of these feature points can be more interpretative and informative compare to other features for symmetry detection. Considering imaging noise and background clutters, the SSM is transformed into Symmetric Transformation Matrix (STM) with the help of the optimized feature matching pairs. STM is a symmetrical matrix in which the symmetric information behind the matched feature pairs could be integrated conveniently and efficiently. Then, the problem of detecting the symmetry axis is converted to mathematical problem of analyzing a symmetrical matrix. Through the STM rank, the reasonable feature matching pairs can be reserved and then the presence/absence of reflection symmetry can be determined.

The main contributions of this paper can be summarized as follows:

1. An idea that taking the task of symmetry axis extraction as a final output of saliency detection is introduced.

2. A refined multi-scale symmetry model based on assigning the salient point symmetry property in the scale-space that adds a region growing method are proposed.

3. A novel feature descriptor to describe the proposed salient symmetry feature.

4. A transfer approach called Symmetric Transformation Matrix (STM) is developed based on feature map to integrate the symmetry information of the image and determine the reasonable potential symmetry axes.

The significance of this work is utilizing the extracted salient symmetry feature for better reflection symmetry axes detection and localization in terms of human attention mechanism. Its application shows that the proposed framework has the potential to help human perceptive and track reflection symmetry object. Experiment results on benchmark datasets also show that the feature presented in this paper is effective and the optimization framework can achieve state-of-the-art performance. 


\section{Materials and Methods}

The pipeline of the proposed framework is shown in Figure 1. For each input image, it starts with searching local extrema in scale-space for generating salient feature points. After filtering the noisy candidates, a set of salient symmetry feature points, i.e., salient symmetry map (SSM), can be got at each scale, and then, a mid-level descriptor which encode these feature point to a feature vector is exploited. Through the feature matching pairs which is generated based on salient symmetry feature description, all the contained symmetric information of these feature pairs in 2-D image plane are integrated in the Symmetric Transformation Matrix (STM) pair by pair. Finally, by analyzing the STM rank, the threshold of a reasonable degree of confidence is calculated,and it will determine all the trust reflection symmetry.

\subsection{Producing salient symmetry feature point}

1). Generating the candidate salient feature point: Considering that SIFT descriptor has utilized extensively due to its robust and effective performance [12], we adopt the method of extracting the local scale-space extrema here, the initial but most crucial step for the SIFT descriptor,to produce the initial salient feature point. Apart from adopting the existing method for convenience, we argue that the local extrema can remain the detail of symmetry object in an image and give the possibility for further optimization. Simply, the first step of detection of salient feature points is obtain the images with different scales. Let $l_{0}$ denotes scale zero of image which is at its original resolution $(1024 \times 768$ same as [34] ). At next scale, the image with half of the original width and height of original image is obtained by down sampling,i.e. $I_{l+1}(x, y)=I_{l}(2 x, 2 y)$. We set 5 scales in the paper totally. At each scale, a set of Gaussian images is generated by blurring the current image through the Gaussian kernel with different standard deviations $\sigma$ (the number of Gaussian images at each scale is 6 , the initial $\sigma=1.60$ and next is $\sqrt{2} \sigma$ ). Using the differences between the Gaussian images, three Difference of Gaussian (DoG) images will have 26 of the pixel values around one pixel( including eight neighbors in the current image and nine neighbors in the above and below respectively), each sample is compared to its all 26 neighbors, if it is extremum, it is candidate salient feature point $s p=(x, y, l)$, where $(x, y)$ is the coordinate of according extreamum. Figure 2(a) illustrates the process of generating DoG in multi-scale space and extracting salient points.

2). Initialize salient symmetry point from candidates: At multi-scale space, on each salient point $s p$ of Gaussian image will be applied by the isotropic symmetry operator. The isotropic symmetry operator has been simply introduced in the Introduction. It is a context-free operator that does not require prior knowledge of the input image, which is originally designed to assign symmetry measure and symmetry orientation to each pixel quantitatively in a local area (usually the size local area is $8 \times 8$ ). Here, we use the isotropic symmetry operator to get salient symmetry point ssp,i.e., the salient point with a high response for symmetry pattern.

Specifically, for each $s p$ at Gaussian image, its symmetry response is calculated based on its surrounding pixels in a kernel (named symmetry kernel, suppose that the symmetry kernel size is $r$ and its optimization details will be covered at next subsection), in other words, for a given salient point which is the center of the determined symmetry kernel, its symmetry response magnitude is determined by all pixel pairs in symmetry kennel, where the pixel pair is set up by two symmetric pixels $\left(p_{i}, p_{j}\right)$, i.e., $s p=\left(p_{i}, p_{j}\right) / 2$. See Figure 2 b.I) for geometric illustration.

With each pixel pair $\left(p_{i}, p_{j}\right)$, we firstly get its intensity gradient by taking the derivative of the image in the horizontal and vertical directions with Sobel filter. Let the horizontal and vertical intensity gradient of one pixel $p_{i}$ are $g_{x, i}$ and $g_{y, i}$ respectively. Then, the gradient magnitude $m_{i}$ and the orientation $\theta_{i}$ is calculated as :

$$
\begin{gathered}
m_{i}=\sqrt{g_{x, i}^{2}+g_{y, i}^{2}} \\
\theta_{i}=\operatorname{atan} 2\left(g_{x, i}, g_{y, i}\right)
\end{gathered}
$$




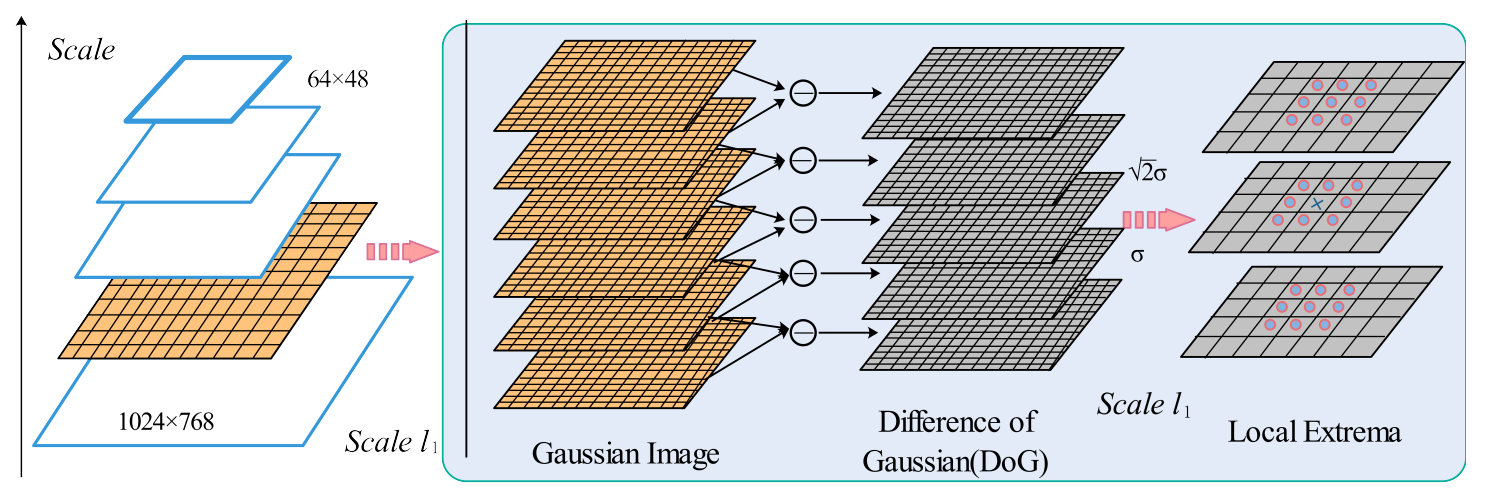

(a) Generating DoG in multi-scale space and extracting salient points, $\ominus$ denotes the difference operation

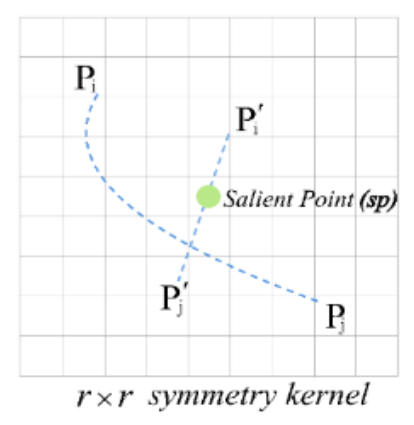

(I) Pixel pairs of salient point

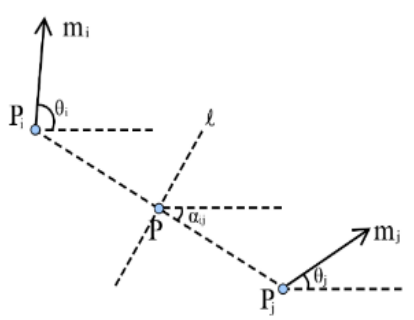

(II) Symmetry orientation of pixel pair

(b) Symmetry pattern of candidate feature point

Figure 2. (a) At each scale, the Gaussian images will be generated,and using the difference of Gaussian image, three DoG images will be got at which the salient point is extracted. (b) I) the $r \times r$ symmetry kernel and the symmetric pixel $p_{i}$ and $p_{j}$. II ). Using the intensity gradients at the pixel locations to calculate contribution of pixel pairs $\left(p_{i}, p_{j}\right)$.

According to the orientation of gradient at point $p_{i}$ and $p_{j}$, an angular symmetry weight of $\left(p_{i}, p_{j}\right)$ can be obtained by function $\omega\left(p_{i}, p_{j}\right)$ :

$$
w\left(p_{i}, p_{j}\right)=\left[1-\cos \left(\theta_{i}+\theta_{j}-2 \alpha_{i j}\right)\right] *\left[1-\cos \left(\theta_{i}-\theta_{j}\right)\right]
$$

Here, $\alpha_{i j}$ is the angle between the line joining $p_{i}$ and $p_{j}$ (let its perpendicular line is $\ell$ ) and the horizontal lines (See Figure $2 \mathrm{~b}$.II).It is easy to find that when the gradient orientations are truly symmetric with $\ell, \theta_{i}+\theta_{j}-2 \alpha_{i j}=\pi$. Therefore, the first term of the function $\omega\left(p_{i}, p_{j}\right)$ has a maximum value at this time. That is consistent with the intuitive notion of symmetry. On the other side, in the situation that the gradient orientations on a straight edge, i.e. $\theta_{i}-\alpha_{i j}=\theta_{j}-\alpha_{i j}=\pi / 2$, the current expression can be compensated by the second term. Based on the Euclidean distance of $\left(p_{i}, p_{j}\right)$, a distance weight of $\left(p_{i}, p_{j}\right)$ is calculated by a Gaussian distance function with a standard deviation $d_{\sigma}\left(p_{i}, p_{j}\right)$ :

$$
d_{\sigma}\left(p_{i}, p_{j}\right)=\frac{1}{\sqrt{2 \pi \sigma}} \exp \left(-\frac{\left\|p_{i}-p_{j}\right\|}{2 \sigma}\right)
$$

It suggests the distance between pixel pairs influences the symmetry measurement of salient point $s p$. The $\sigma$ corresponds to the standard deviations of current Gaussian image.With the angular symmetry 
weight $w\left(p_{i}, p_{j}\right)$ and distance symmetry weight $d_{\sigma}\left(p_{i}, p_{j}\right)$ of $s\left(p_{i}, p_{j}\right)$, the contribution of single pixels pair to local symmetry response of given salient point $s p$ is calculated by the function $s\left(p_{i}, p_{j}\right)$ :

$$
s\left(p_{i}, p_{j}\right)=d_{\sigma}\left(p_{i}, p_{j}\right) \cdot w\left(p_{i}, p_{j}\right) \cdot \log \left(1+m_{i}\right) \cdot \log \left(1+m_{j}\right)
$$

where $m_{i}$ is the magnitude of the gradient, the multiplication with the gradient magnitudes ensures that salient points response to high local symmetry value and logarithms are used to reduce the influence of large numerical equivalence. Finally, the symmetry response of the salient point $s p$ can be got by linearly summing the contribution of all pixel pairs in the symmetry kernel, i.e.

$$
M_{\sigma}^{r}(s p)=\sum_{(x, y) \in \Gamma(s p)} s(i, j)
$$

$\Gamma(s p)$ is the symmetry kernel region and $r$ is its size.

We argue that the salient points with a high symmetry response are positive for detecting the symmetry pattern at the image level. So we set a threshold $t$, and only the salient point with symmetry response magnitude greater than $t$ are preserved as salient symmetry point $s s p=(x, y, l)$. The final collection of salient symmetry points at scale $l$ refers to the salient symmetry feature map $\left(S S M_{l}\right)$. Considering the needs of getting enough high-quality candidate points, an empirical formula is set manually to determine the value of $t$ according to different standard deviations $\sigma$ :

$$
t=0.8-(0.15 * \sigma)
$$

3). Adaptive Kernel Size algorithm: Hereto, the salient symmetry point $s s p=(x, y, l)$ of an image can be generated as follow: Given an image, it is firstly downsampled to get the multi-scale images, at each scale, the current image is blurred by different Gaussian kernels, and with DoG, the salient point is extracted by detecting the local extrema. Then, at each scale, the symmetry response of salient point is calculated and those who with high symmetry response are preserved as final salient symmetry feature points.

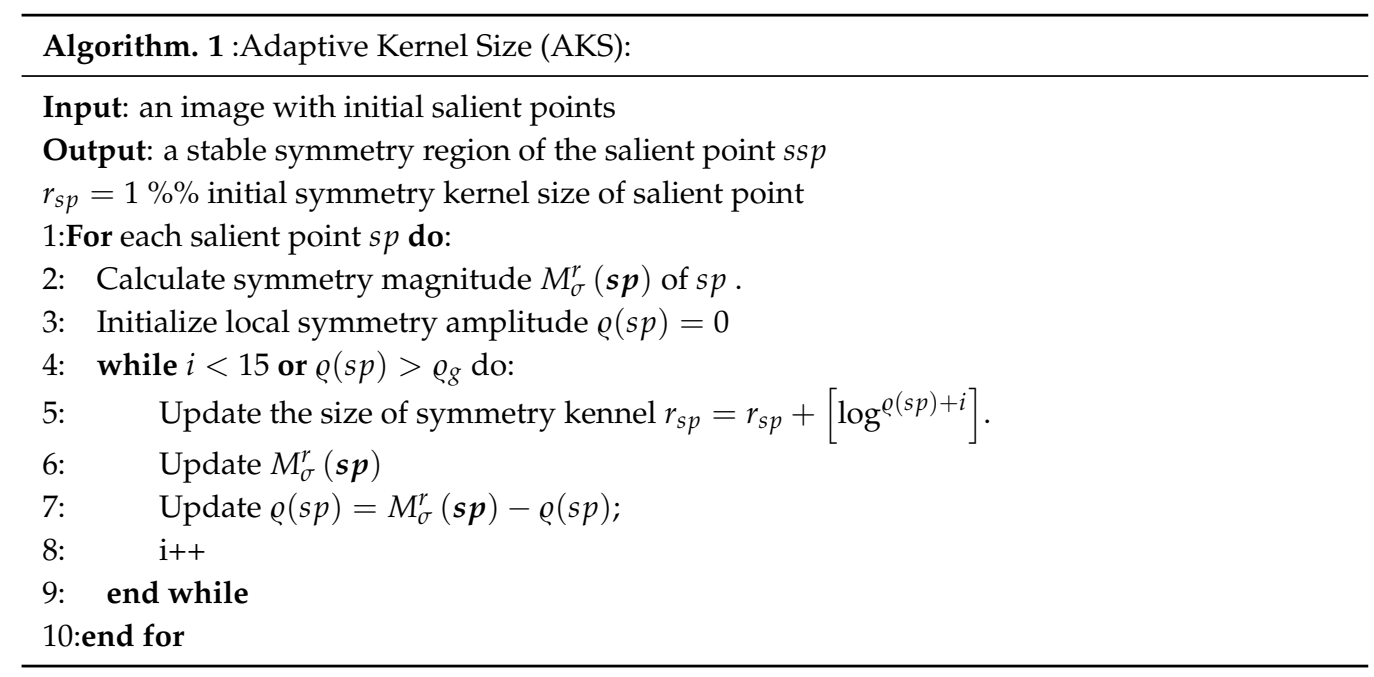

Nevertheless, there is one quite important problem is ignored — the symmetry kernel size. obviously, for images with unknown information, the size of symmetry kennel is crucial. The size of the symmetry kernel is smaller, the loss of local information makes the symmetry response of the salient point less measured, resulting in filtering away some important features. When it is larger, the more symmetry pattern of salient point would be detected in theory, but it also comes with some problems. Firstly, quite a few pixel pairs are far from the center, leading these pixel pairs to have an extremely low distance weight which doesn't make sense to calculate symmetric measurement values. 
Secondly, the larger symmetric kernel means more image information need to be computed, which greatly increases the computational load of the algorithm.

To address this problem, we develop an Adaptive Kernel Size (AKS) algorithm 2.1, which is a region-growing algorithm. The symmetry kernel region of $s p$ starts from the salient point itself and grows gradually by including its neighboring pixels that have the same sym-center. The growing process is iterative until the stable local symmetry region of the $s p$ is formed, in other words, the expansion of a region will stop if the symmetry response of $s p$ not increase. Let $\varrho(s p)$ denotes the growing criterion, the region ends growing when $\varrho(s p)<\varrho_{g}$. The rationale behind the equation is that the salient point of the updated symmetry kernel has the highest symmetry response and has been independent with the size of the local area. In this work, $\varrho(s p)$ refers to the local symmetry amplitude of $s p$ responding to symmetry pattern of current symmetry kernel, and $\varrho_{g}$ refers to a convergent value (we set $\varrho_{g}=0.1$ in our experiment). This threshold can be tuned to allow more or less tolerance to the expansion criterion, resulting in different kernel sizes. When the local symmetry amplitude is convergent, the symmetry kernel is best to respond to the potential symmetric structure of this salient point. In order to accelerate the convergence speed and avoid falling into local optimal value, we set up a rough formula $r=r+\left[\log ^{e(s p)+i}\right]$ to update the size of symmetry kernel, where the growth rate of size can be adjusted according to the actual situation. Owing to the limitation of the iteration $i$, on the other hand, it is possible to avoid the over-speed of the growing rate and the error of the optimal value.

\subsection{Feature Description and Feature matching}

1) Salient symmetry feature descriptor:To computer a feature descriptor for each salient symmetry point $s s p$, we use a strategy similar to Orientation Assignment process in [31] and that of shape context[35] to encode the salient feature point salient symmetry feature(SSF) vector: Let the feature descriptor $D_{s s p_{i}}=\left\{\theta_{i}, \delta_{i}, \kappa_{i}\right\} \subseteq \mathbb{R}^{3}, s s p_{i} \in \mathbb{R}^{4}, \forall i \in\{1,2, \ldots, n\}$, consists of three different components: direction component $\theta$, scale invariant component $\delta$ and adaptive kernel size $\kappa$. Direction component $\theta$ measures the spatial information of a ssp, it formed by a vector which contains all the gradient orientation histogram in a predetermined region that around the keypoint through Orientation Assignment process. $\theta=\left\{\theta_{i}\right\}_{i=1}^{n}, \theta_{i} \in \mathbb{R}^{128}$ is 128 -dimension and is rotation invariant due to the rotation of gradient orientations by the dominant orientations of the key points. Scale invariant component $\delta=\left\{\delta_{i}\right\}_{i}^{n}$ contains the scale information of a ssp, where $\delta_{i} \in[0,5]$ coincides with [16],corresponding to scales of multi-scale salient symmetry map. The adaptive kernel size $\kappa$ of the $s s p$ is also important local-based feature, which measures the stability of the symmetry structure. Three equal and independent components result in a 130-dimensional descriptor.

2) Matching SSF of original image with mirrored one: With salient symmetry feature,the original image and its flipped version can be matched for finding symmetrical salient feature point of an object. Let $\left\{D_{s s p_{i}}\right\}_{i=1}^{n}$ is the set of descriptors for $n s s p$ of original image, and $\left\{D_{s s p_{i}}^{m}\right\}_{i=1}^{n}$ is the mirrored descriptors of $s s p_{i}^{m}$, where $s s p_{i}^{m} \in\left\{s s p_{i}^{m}\right\}_{i=1}^{n}$ is set of the salient symmetry points in the flipped original image. See Figure 3(a) for illustrating the process of finding candidate pairs of mirror symmetric points. The salient symmetry point $s s p_{i} \in\left\{s s p_{i}\right\}_{i=1}^{n}$ and salient symmetry point of the mirrored image $s s p_{k}^{m} \in\left\{s s p_{i}^{m}\right\}_{i=1}^{n}$ can form a candidate pair of mirror symmetric points $s s p_{i, k}^{m}=\left(s s p_{i}, s s p_{k}^{m}\right)$ calculated by given equation below:

$$
s s p_{i, k}^{m}=\arg \min \left\|D_{s s p_{i}}-D_{s s p_{k}}^{m}\right\|_{2}
$$

Then the $s s p_{k}$ of original image can match with $s s p_{i}$ to form a pair of candidate mirrored reflection symmetry pair at original image. Aim to eliminate the noise error and detect reflection symmetry point more accurately, inspired by Random Search Scheme in [23], the neighbors of $s s p_{k}$ are considered to update the matched feature point of $s s p_{i}$. See Figure $3(\mathrm{~b})$ : around $s s p_{k}, e$ salient feature points are randomly selected in the circular region of radius $\lambda$. Let $\left\{R_{\lambda}\right\}_{1}^{e}$ is set of neighbors of $s s p_{k}$, where $R_{\lambda}$ 


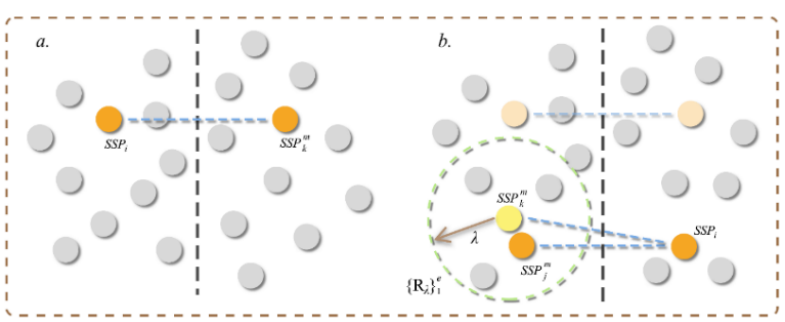

Figure 3. The dots on the left side of dotted line represent the feature points of the original image,and that on the other side represents the feature points of the flipped image. (a) $\left(s s p_{i}, s s p_{k}^{m}\right)$ is mirror symmetric points. (b) Random Search Scheme. Let the neighbors of $s s p_{k}^{m}$ update the mirror reflection point of $s s p_{i}$.

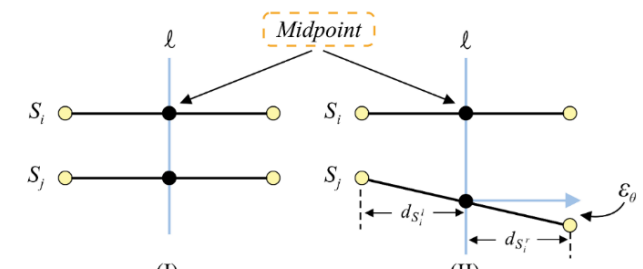

(I)

(II)

Figure 4. $S_{i}$ denotes $i$-th pair of matched $s s p_{j}$ and $s s p_{k}$ which is connected by a line. (I) a line $\ell$ is perpendicular to these parallel lines. (II) If $S_{j}$ satisfies the angular tolerance and distance tolerance, it supports $\ell$ which is determined by $S_{i}$,

is circular region of radius $\lambda$ ((we set $e=8$ and $\lambda=10$ ). The best mirror marched point from the neighbor collection is updated as given below which is similar to equation 8 :

$$
\begin{aligned}
& s s p_{i, j}=\arg \min \left\|D_{s s p_{i}}-D_{s s p_{j}}^{m}\right\|_{2} \\
& s s p_{j} \in\left\{R_{\lambda}\right\}_{1}^{e}
\end{aligned}
$$

Though adds a little bit of computational complexity, Random Search step promotes the current estimate of the mirror reflection of a point. It is an essential part of symmetric matching pairs and affects the next steps of the algorithm directly. Note that it would not be better for some point because neighbors would be not going to perform better in some cases.

\subsection{Symmetric Transformation Matrix (STM) for reflection symmetry axis}

1) Constructing Symmetric Transformation Matrix: The construction of Symmetric Transformation Matrix is based on matched mirror reflection pairs. Let $S=\left\{s s p_{i, k} i, k \in\{1,2, \ldots n\}\right\}_{i=1}^{p}=\left\{S_{1}, S_{2}, \ldots, S_{p}\right\}$ be the set of detected $p$ pairs of matched feature points. The motivation of construction of STM comes from the geometric fact that in a parallel scenario, if a vertical line is perpendicular to a line, it will be perpendicular all lines of a set of parallel lines which the line belongs to (see Figure 4 (I)), in other words, a set of parallel lines would be bisected by one vertical line. Then we can derive that a line joining a pair of matched mirror points will determine a vertical line that can be a potential symmetry axis of the image, passing through the midpoint.

In the above work, the generated matched featured pairs can be viewed as a set of lines connecting feature points at both ends, and there will be a set of determined vertical lines. Therefore, the task of detecting the symmetry axis can be converted into a probabilistic problem that the perpendicular determined by a pair of matched feature points to what extent is accepted by an image as the symmetry axis of that image. To address this problem, it needs to verify how many parallel lines support that line. In this case, different from the voting-based scheme, we construct the (STM) to calculate the support rate of the vertical line and see it as the probability of that line becoming the symmetry axis of an image.

Let $M_{p \times p}$ is our STM, where $p$ corresponds to the number of matched feature pairs. Each element $M_{i, j}$ of $M$ is determined by the quality of two matched feature pairs $S_{i}$ and $S_{j}$ as follows:

$$
M_{i j}= \begin{cases}1 & S_{j} \in M S_{i} \text { or } i=j \\ 0 & S_{j} \notin M S_{i}\end{cases}
$$




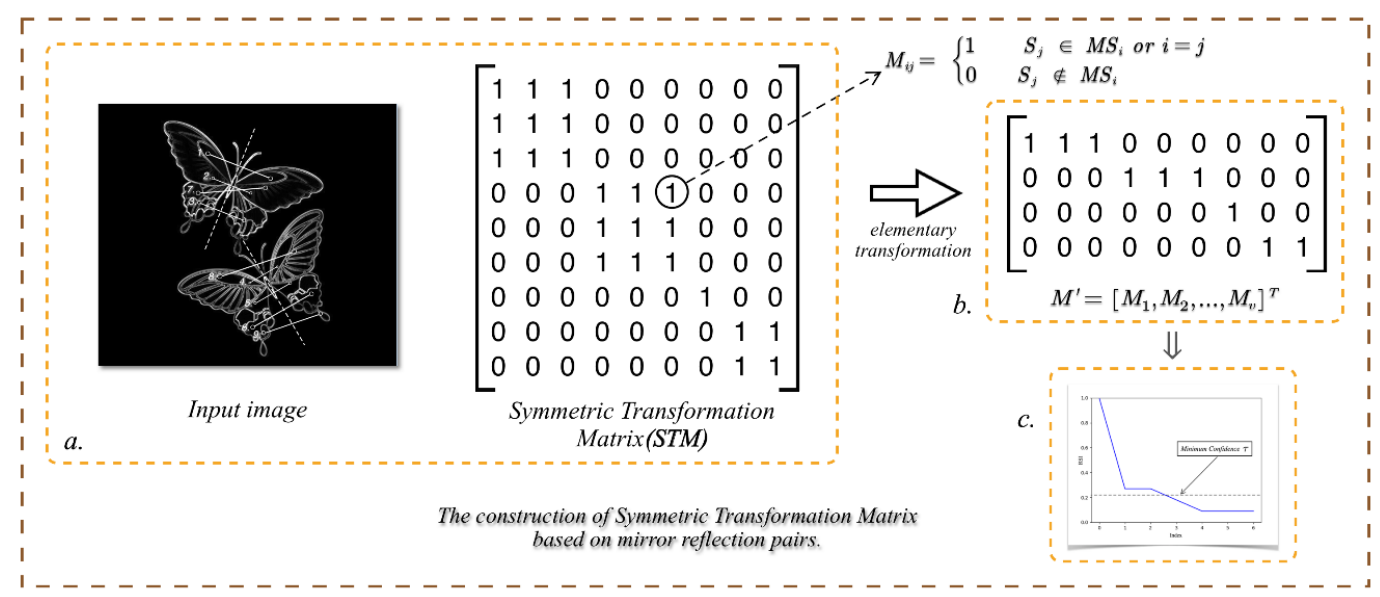

Figure 5. a) The construction of STM is based on reflection feature pairs. In the input image, mirror reflection pairs 1,2 and 3 support a symmetry axis, and the same to 4,5,6 and 8,9. Only mirror reflection pairs 7 determine a symmetry axis alone which no one other mirror reflection pairs agree with. b) $M^{\prime}$ is the rank matrix that gets rid of repeated rows of STM after elementary transformation. c) a confidence curve versus minimum confidence $\tau$ to determine reasonable axes present in the image

Here, $M S_{i}$ is set of matched feature pairs which support the vertical line determined by $S_{i}$, i.e. $M S_{i}$ is a collection of matched feature pairs support the same vertical line $S_{i}$, and we define a pair of matched feature pairs $S_{j}$ support $\ell_{i}$ if it satisfies the following equation :

$$
\arccos \frac{\overrightarrow{S_{i}} \cdot \overrightarrow{S_{j}}}{\left\|\overrightarrow{S_{i}}\right\|\left\|\overrightarrow{S_{j}}\right\|}<\varepsilon_{\theta} \text { s.t. }\left\|\overrightarrow{d_{S_{j}} \ell_{i}}\right\| \in(0,0.05)
$$

The constraint $\left\|\overrightarrow{d_{S_{j}}} \ell_{i}\right\|$ is the distance of the midpoint of $S_{j}$ from the line $\ell_{i}$, which ensures that $S_{i}$ and $S_{i}$ are on a vertical channel. $\varepsilon_{\theta}$ is a notion that we adapt from [28] which represents the angular tolerance parameter, it is key to measure if $S_{j}$ belongs to $M S_{i}$. See Figure 4(II) for a graphical illustration. Experimentally we set $\varepsilon_{\theta}=2^{\circ}$.

As the matched feature pairs are encoded to a matrix, the symmetry information behind these feature pairs is then integrated in STM with a binarization form, which saves us from the intricate background and noise of the image and focuses on analysing the STM. A simple transformation is shown in Figure 5(a).

2) Detecting reasonable reflection symmetry axis: Each element represents a pair of matched feature points, it is obvious that the constructed STM is a symmetric matrix due to transmissibility of parallel lines. In the best situation, STM is an identity matrix which the rank $v$ is 1 , it means the all the matched feature pairs support one vertical, while in a bad situation,i.e., there is no any symmetry pattern in an image, STM will be a diagonal matrix which rank is 0 .

According to the process of calculating the rank of the matrix, it is easy to find that the value of $v$ is also the amount of potential symmetry axis and $v<=p$. The reason why there so many repeated rows in STM is because there are many mirror reflection pairs agree or approximately agree with the same vertical line. Let $M^{\prime}$ is a rank matrix that gets rid of repeated rows of STM after elementary transformation and the number of rows of a matrix $M^{\prime}=\left[M_{1}, M_{2}, \ldots, M_{v}\right]^{T}$ corresponding to the rank $v$ (see Figure 5(b)). Row vector $M_{i} \in M^{\prime}$ mean $i$-th potential axis of symmetry determined by $i$-th mirror reflection pairs.

Let $\ell_{i}$ denotes the vertical line corresponding to $M_{i} \in M^{\prime}$. Ideally, each $\ell_{i}$ would be a potential symmetry of image as there would be multiple symmetric objects in an image. Nevertheless, not each $\ell_{i}$ is trustworthy. Thus, we define a cost function to measure how far the potential axis reflection symmetry axis of the image is from the ground-truth: 

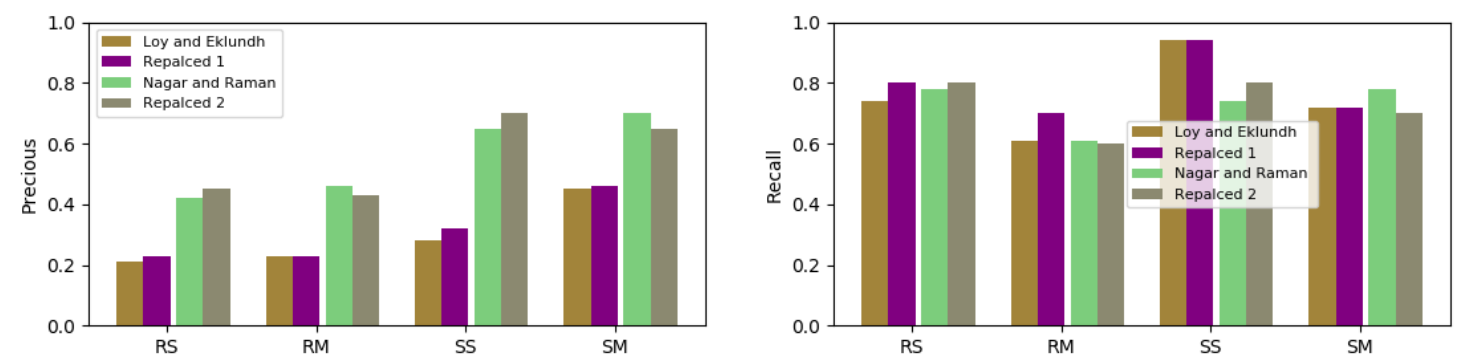

Figure 6. Proposed feature in two feature-centered algorithms: Loy and Eklundh 's [12], Nagar and Raman 's [30]. RS is single symmetry images, RM is multiple axis images;SS and SM refer to single axis and multiple axes of synthetic image respectively

$$
J\left(\ell_{i}\right)=\frac{1}{p} \sum_{M_{j} \in M^{\prime}} \sum_{k=1}^{p} M_{i k} \oplus M_{j k}, i \in[1, v]
$$

Since STM is composed of binary vectors, $\oplus$ here denotes a exclusive or operator which is defined that if two variables are equal, the result is 1 , otherwise 0 . The cost function measures the cost at which a line determined by a matched feature pair is supported by all the matching pairs. The higher the cost, the more acceptable the axis of symmetry is. To get a better result, a minimum confidence score $\tau$ (see Figure 5(c)) is defined to filter untrusted axis:

$$
\tau=\frac{1}{v} \sum_{i=1}^{v} J\left(\ell_{i}\right)
$$

The value of $\tau$ is iteratively varying with $j\left(\ell_{i}\right)$, which ensures that every trust symmetry axis is not missed. When $j\left(\ell_{i}\right)>\tau$, the candidate axis $\ell_{i}$ will be accepted. Here, the parameters of each symmetry axis are not given, because if the line is determined, and the parameters can be determined accordingly.

\section{Results and Evaluation}

This section presents the experiments evaluating the salient symmetry feature strategy against state-of-the-art feature-centered methods and validating the effectiveness of our framework for reflection symmetry axis detection.

\subsection{Dataset}

The feature strategy proposed in this paper is evaluated by comparing it with state-of-the-art feature-centered methods [12] and [30]. To ensure consistency of datasets, it has tested on the standard dataset (Ds1) provided by [36] which contains 258 images in two categories: 164 real images where 79 single symmetry images (RS) and 85 multiple axis images(RM); 94 synthetic images where 55 single axes (SS) images and 39 multiple axes (SM), respectively.

The detection framework has tested and evaluated on the benchmark dataset (Ds2) in [37]. Ds2 is the updated version of [38] in 2017, containing images with a single reflection symmetry as well as multiple symmetries.

\subsection{Evaluation of Salient Symmetry feature map}

To evaluate the performance of the salient symmetry feature proposed, the SIFT descriptor used in the selected two typical feature-centered algorithms are replaced with the developed feature scheme proposed in Section 3. The standard precision-recall involved in [37] is used as quantitative evaluation metrics. Precision measures the accuracy of each test, it represents the number of true positives: $\mathrm{P}=\mathrm{TP} /(\mathrm{TP}+\mathrm{FP})$. The recall is a measure of completeness, it counts the number of ground-truth 

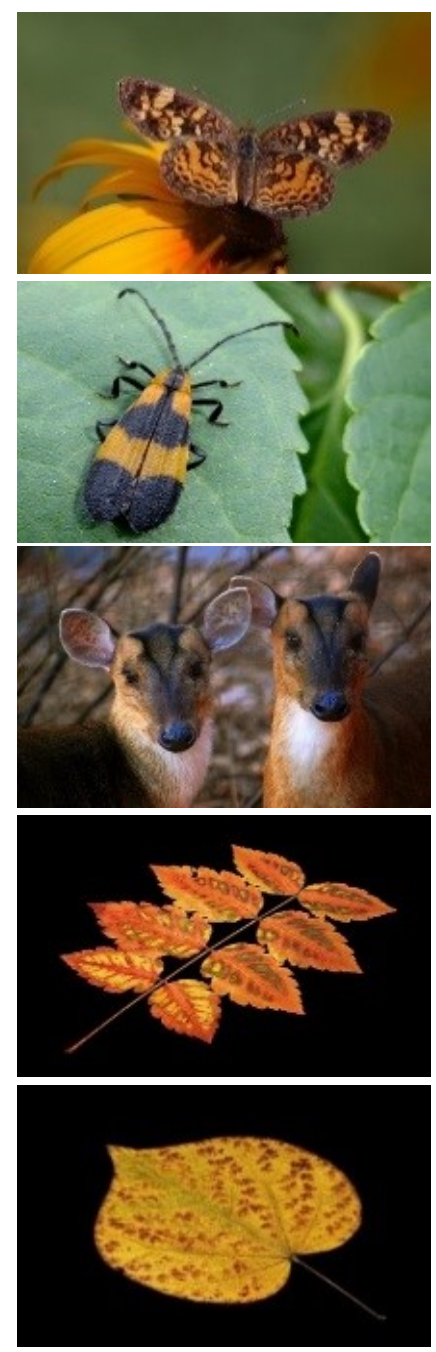

(a)
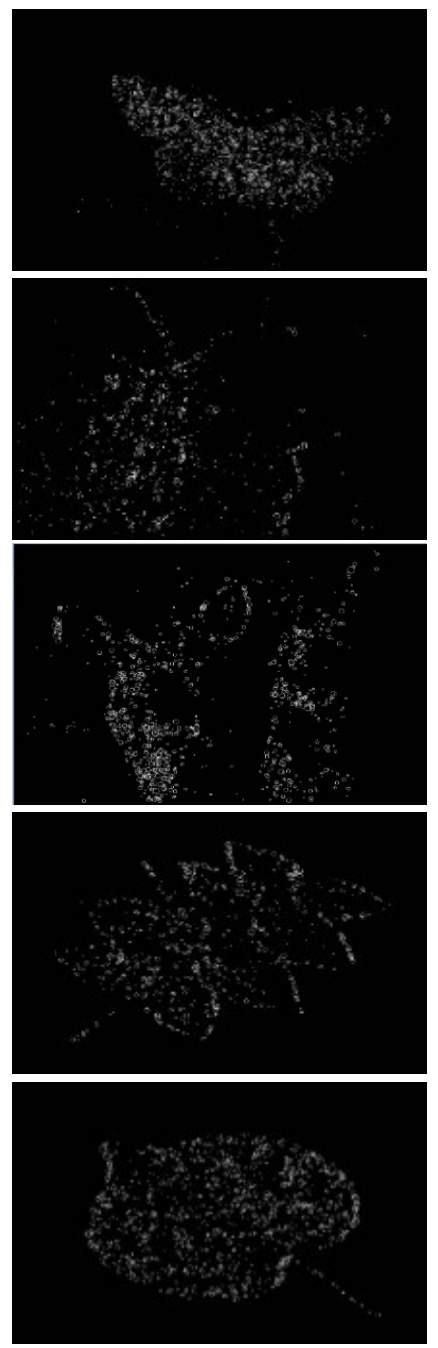

(b)
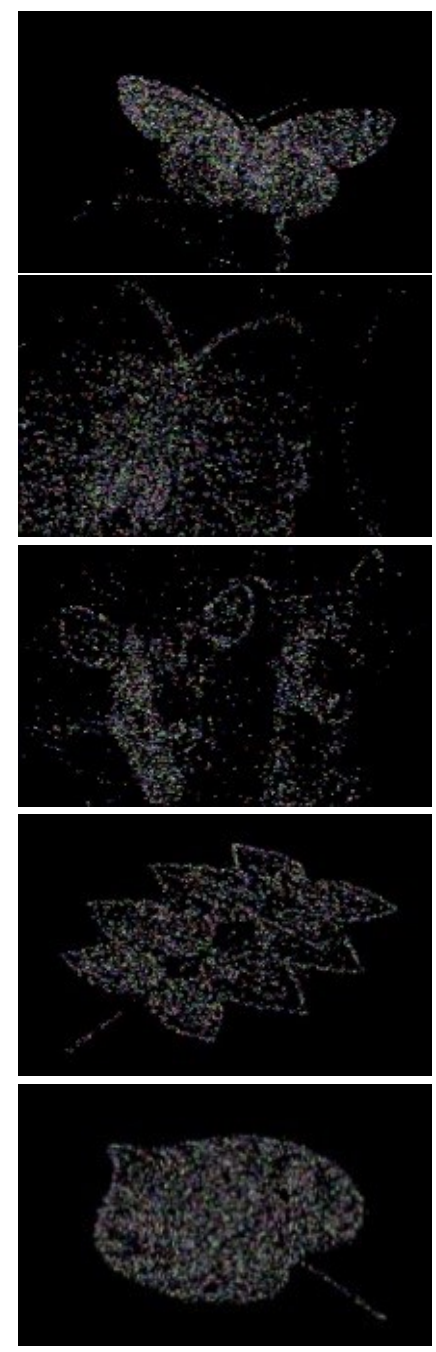

(c)

Figure 7. (a) Input image. (b) Salient symmetry features maps of the input images. (c) SIFT maps.

positives: $\mathrm{P}=\mathrm{TP} /(\mathrm{TP}+\mathrm{FN})$. Here, $\mathrm{TP}, \mathrm{FP}, \mathrm{FN}$ denote the number of the detected correct axis, the wrong axis in the detected group, and the error-detecting axis respectively. For the evaluation of reflection axis detection, the angle tolerance $\theta_{t}[38]$ between the detected $l_{d}$ and ground-truth axis $l_{G t}$, and the distance tolerance $d_{t}$ [38] between the centers of these two lines need to be calculated. The detected axis is correct if $\theta_{t}$ and $d_{t}$ is no more than the predetermined threshold $t 1$ and $t 2$ respectively, where $t 1=2^{\circ}$ and $t 2=0.2 \times \min \left(l_{G T}, l_{d}\right)$ according to [38]. Figure 6 shows the results of precision and recall values. When the replaced feature used in Loy's methods[12], its effect has been improved slightly in the case of a single axis while relatively obviously for real images with multiple axes. By contrast, the performance of replaced feature in [30] raised more than in [12]. However, the performance decreased compared with the performance of the original feature when it comes to the multiple symmetry, it might because the SIFT features further optimized in prophase of [30], which filtered the symmetry pairs that didn't match each other, leading our features do not have a distinct advantage in this algorithm. It is also possible that the information ignored in the keypoints processing strategy is responsible for making the matching scheme of [30] cannot reach the optimal value.

According to the multi-scale fusion method of [16] and [25], after determining multi-scale salient symmetry maps, we combine these maps to same scale $(l=2)$ for displaying our feature points. Figure 7 shows the combined salient symmetry map Figure 7(b) with the initial region growing threshold $\varrho_{g}$ for five example images from the dataset Ds1. Comparing with SIFT Figure 7 (c), the 


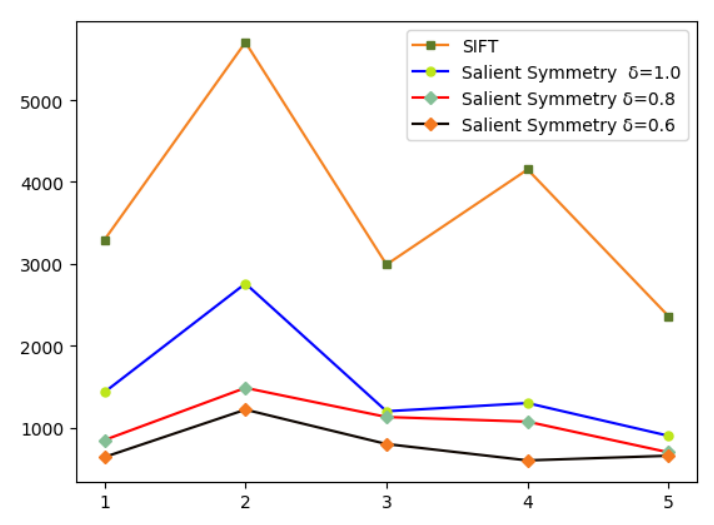

Figure 8. Comparisons of keypoints with different threshold and The SIFT method.

number of salient symmetry point is less than that of SIFT interesting point. Figure 8 gives quantitative comparisons of keypoints of SIFT with the salient symmetry points on different $\varrho_{g}$. It is clear that as the $\varrho_{g}$ increases, the figures of keypoints information of the proposed for these examples rise as well. Also, as Figure 7 (b) shows that the contour information which reflects visual symmetry of symmetrical objects is largely retained, even though the key point of the proposed method are relatively sparse compared with SFIT's.

Meanwhile, owing to background clutter, there are still some outliers in the salient symmetry map that can be seen above. Here, outliers are defined as points scattering among the image background and not focusing on the symmetry objects. Actually, these outliers are all initial salient points that are not filtered. We argue that the reason why there are existing outliers is that the noise of the asymmetric region is not much different from the real symmetry information on the local scale. The existing outlier is a trade-off of better performance. By limit the value of $\varrho_{g}$ or increasing the salient symmetry point threshold $t$, the number of outliers can be decreased, but the quantity of useful salient symmetry point decline as well.

\subsection{Evaluation of Reflection Symmetry Axes Detection}

The axis detection framework is tested on the dataset Ds2. Figure 9 and Figure 10 show the examples of detected single axis and multiple axes respectively. The matched feature pairs are connected in a random color, the detected axes are red and the minimum confidence score $\tau$ is also reported below each sample image. It can be observed that the value of $\tau$ in multiple axis images is relatively lower than that in the single-axis case, that is because the existing other one axis will affect the support of the feature matching pair to the one axis. Also because of that, only one axis of multiple axes in an image is detected.

Table 1. Highest F-measure for single and multiple cases

\begin{tabular}{|c|c|c|c|c|}
\hline Approaches & Loy et al.[12] & Elawady et al. [19] & Nagar et al.[39] & Proposed \\
\hline Single F & 0.45 & 0.40 & 0.68 & $\mathbf{0 . 4 8}$ \\
\hline Multiple F & 0.30 & 0.22 & 0.20 & $\mathbf{0 . 2 5}$ \\
\hline
\end{tabular}

The proposed optimization framework is compared with state-of-art approaches $[12,19,39]$ on dataset Ds2. The source codes of [19] and [39] are not available, but the precision versus recall curves for the methods are available which is given in the ICCV challenge competition report [36], and it also can be seen in [39]. The report of the precision versus recall curves can be seen in Figure 11, it can be observed that the proposed framework can reach the second-highest performance both for the single and multiple cases but without a obvious gap with the best method [39]. For reflection symmetry 


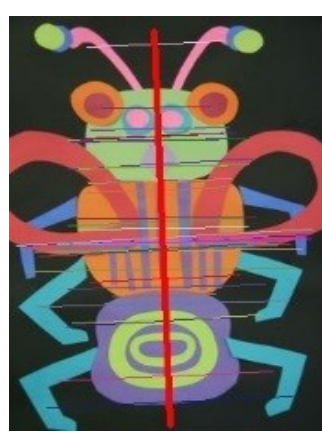

(a) $\tau=0.350$

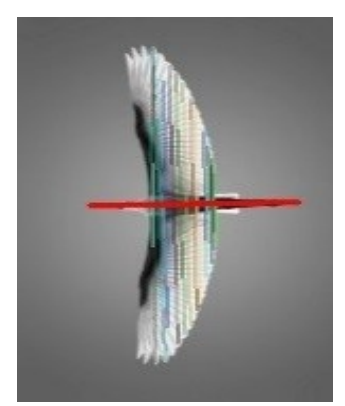

(b) $\tau=0.620$

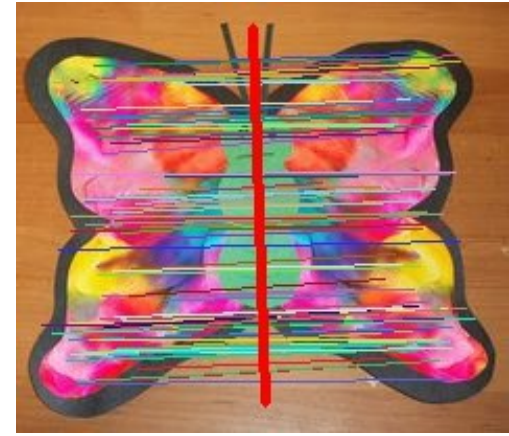

(c) $\tau=0.100$

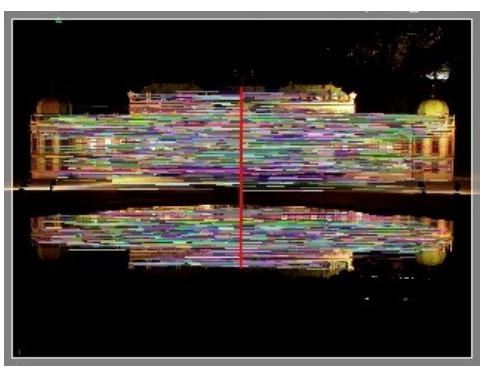

(d) $\tau=0.455$

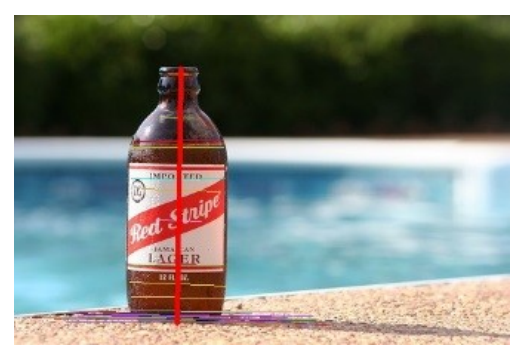

(e) $\tau=0.200$

Figure 9. Results of single axis. The feature matching pairs are connected in random color and the detected axes is red

with a single axis, the method beats other approaches except for [39]. For multiple axes situations, the proposed method achieves the state-of-art performance [39] which also shows its competitiveness.

The single value used in [37] named F-measure is also adopted as a metric for further comparison. Table 1 shows the highest F-measure. Compare with Loy's method, our F-measure for multiple axes is 0.10 less but 0.03 better for a single axis, that is probably because the disturb of outliers in our method decrease the performance of single axis detection, while the existing of STM makes up for the deficiency of Loy'method that it does not put forward a clear optimization plan for multiple axes detection.

\section{Conclusion and Future Work}

In this paper, an optimization framework embedded with a salient symmetry feature is proposed for the detection of reflection symmetry axes of an image. The salient symmetry feature is a description of a set of salient symmetry points in visual saliency, which preserves more details in the symmetric regions of an image. The Symmetric Transformation Matrix, on basis of optimized feature matching pairs, integrates all symmetric matched pairs information in form of binarization matrix. Through the STM itself and its rank, the confidence of the potential symmetry axis is determined, helping to detect all potential symmetry axes. Verified on the standard dataset, the results show that the proposed salient symmetry feature can help the existing method make improvements. The framework is also investigated, which shows that even though the presented framework only achieves the second highest performance from a comprehensive metric, it has its advantages compared with the state-of-art method. However, it shows that the salient symmetry point still has some points outside of symmetric objects because the image is smooth, and Symmetric Transformation Matrix fails to detect the symmetry axes without accurately matched feature pairs. As for future work, we would like to develop an advanced strategy with multiple possibilities to achieve more robust performance and attempt to derive better mathematical expressions to optimize parameters. Other computer vision technologies are also under consideration for further symmetry detection. 


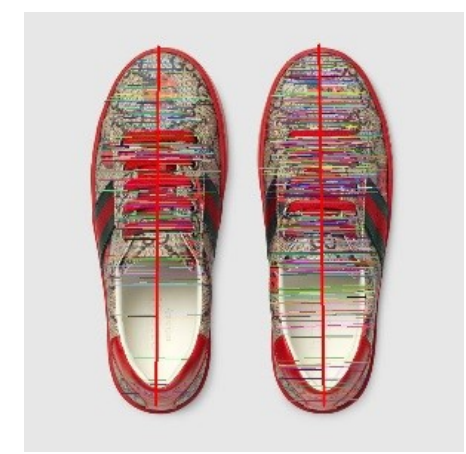

(a) $\tau=0.190$

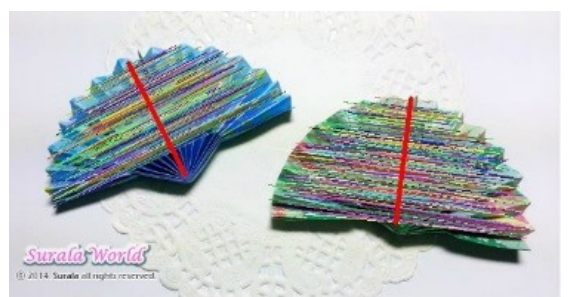

(b) $\tau=0.130$

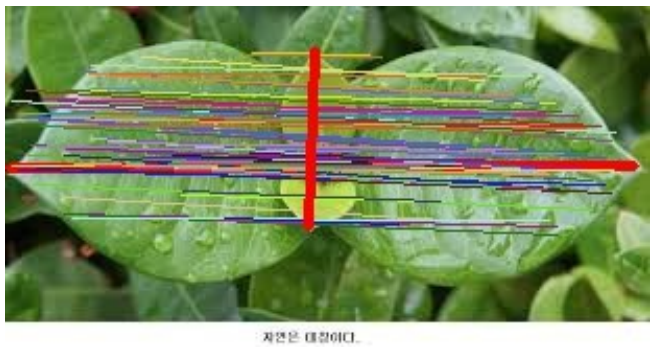

(c) $\tau=0.430$

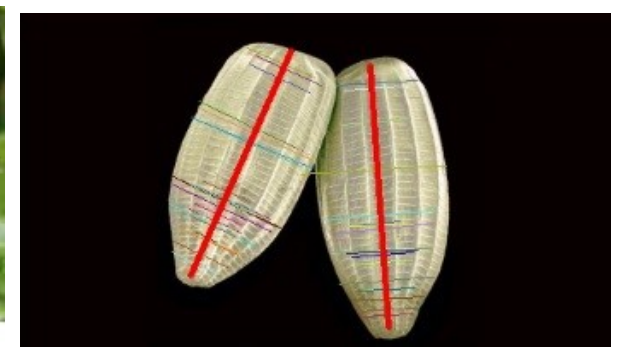

(d) $\tau=0.100$

Figure 10. Results of multiple axis.

Author Contributions: For research articles, the conceptualization, methodology, software, formal analysis, investigation, resources, writing - original draft preparation were performed by K.W., validation and visualization were executed by Y.Q., and M.J. were responsible for writing-review and editing. The supervision, project administration and funding acquisition were contributed to the work by D. J. and C.L. All authors have read and agreed to the published version of the manuscript.

Funding: This work was supported by National Natural Science Foundation of China $(61902232,61902231)$, Natural Science Foundation of Guangdong Province (2019A1515010943), Key Project of Basic and Applied Basic Research of Colleges and Universities in Guangdong Province (Natural Science) (2018KZDXM035), the Basic and Applied Basic Research of Colleges and Universities in Guangdong Province (Special Projects in Artificial Intelligence)(2019KZDZX1030), 2020 Li Ka Shing Foundation Cross-Disciplinary Research Grant (2020LKSFG04D).

Acknowledgments: The authors would like to thank anonymous reviewers for their very detailed and helpful review.
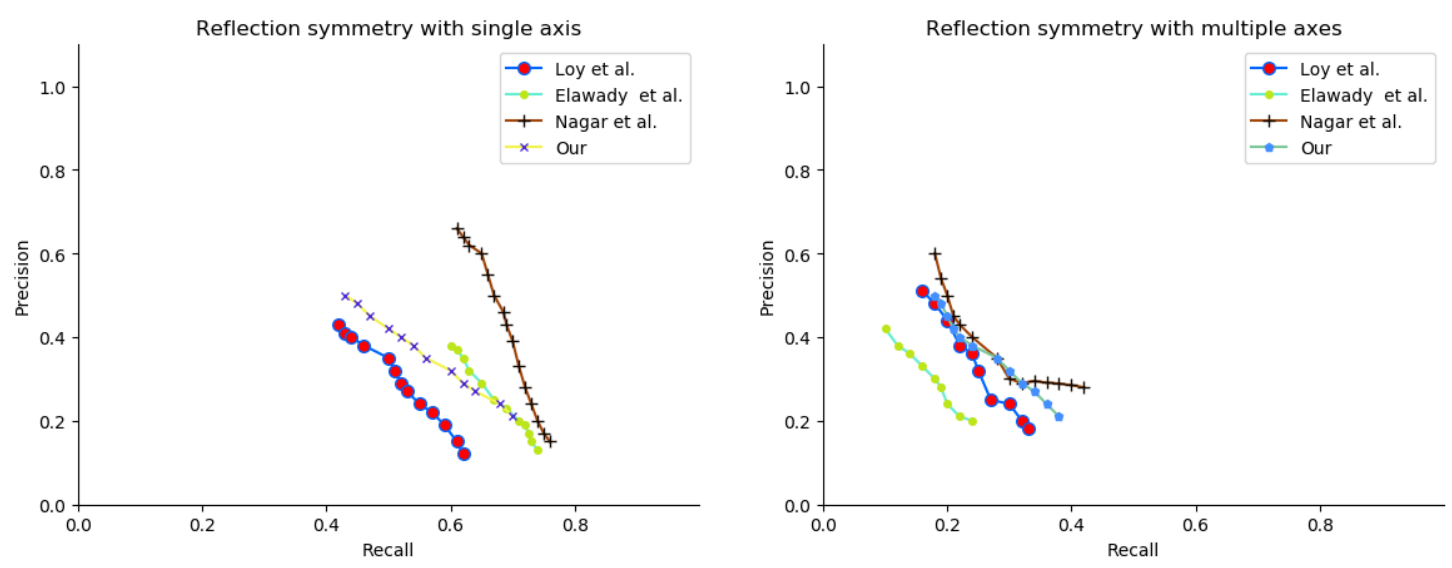

Figure 11. Precision versus recall curves. 
Conflicts of Interest: The authors declare no conflicts of interest. The funders had no role in the design of the study; in the collection, analyses, or interpretation of data; in the writing of the manuscript, or in the decision to publish the results.

\section{References}

1. Mitra, S.; Liu, Y. Local facial asymmetry for expression classification. Computer Vision and Pattern Recognition, 2004. CVPR 2004. Proceedings of the 2004 IEEE Computer Society Conference on, 2004.

2. Tyler, C.W. Human Symmetry Perception and Its Computational Analysis; 2002.

3. Kim, V.G.; Lipman, Y.; Funkhouser, T. Symmetry-guided texture synthesis and manipulation. Acm Transactions on Graphics, 31, 1-14.

4. Koser, K.C.Z.; Pollefeys., M. Dense 3D Reconstruction of Symmetric Scenes from a Single Image. Joint Pattern Recognition Symposium 2011.

5. Sela, G.; Levine, M.D. Real-Time Attention for Robotic Vision. Real-Time Imaging, 3, 173-194.

6. Janidarmian, M.; Roshan Fekr, A.R.K.Z.Z. A Comprehensive Analysis on Wearable Acceleration Sensors in Human Activity Recognition. Sensors, 17.

7. Dan, R.; Bronstein, A.M.; Bronstein, M.M.; Kimmel, R. Full and Partial Symmetries of Non-rigid Shapes. $89,18-39$.

8. Mitra, N.J.; Pauly, M.; Wand, M.; Ceylan, D. Symmetry in 3D Geometry: Extraction and Applications. Computer Graphics Forum 2013, 32, 1-23.

9. Mukhopadhyay A., S.B.; Porikli., F. Detection and characterization of Intrinsic symmetry of 3D shapes. International Conference on Pattern Recognition., 2017.

10. Cicconet, M.; Birodkar, V.; Lund, M.; Werman, M.; Geiger, D. A convolutional approach to reflection symmetry. Pattern Recognition Letters, p. S0167865517300971.

11. Kootstra, G.; Jong, S.D.; Schomaker, L.R.B. Using Local Symmetry for Landmark Selection 2009. 5815, 94-103.

12. Loy, G.; Eklundh, J.O. Detecting Symmetry and Symmetric Constellations of Features; 2006.

13. Reisfeld, D.; Wolfson, H.; Yeshurun, Y. Context-free attentional operators: The generalized symmetry transform. International Journal of Computer Vision, 14, 119-130.

14. Pritts, J.; Chum, O.; Matas, J. Rectification, and Segmentation of Coplanar Repeated Patterns. 2014 IEEE Conference on Computer Vision and Pattern Recognition (CVPR), 2014.

15. Sun, Y.; Bhanu, B. Reflection Symmetry-Integrated Image Segmentation. IEEE Trans Pattern Anal Mach Intell, 34, 0-0.

16. Kootstra, G.; de Boer, B.; Schomaker, L.R.B. Predicting Eye Fixations on Complex Visual Stimuli Using Local Symmetry. 3, 223-240.

17. Verma, A.; Van der Haegen, L.; Brysbaert, M. Symmetry detection in typically and atypically speech lateralized individuals: A visual half-field study. Neuropsychologia, 51, 2611-2619.

18. Zhao, S.; Gao, Y.; Jiang, X.; Yao, H.; Chua, T.S.; Sun, X. Exploring Principles-of-Art Features For Image Emotion Recognition 2014.

19. Elawady, M.; Ducottet, C.; Alata, O.; Barat, C.; Colantoni, P. Wavelet-Based Reflection Symmetry Detection via Textural and Color Histograms. 2017 IEEE International Conference on Computer Vision Workshop (ICCVW), 2017.

20. Herbert, B.; Andreas. Speeded-Up Robust Features (SURF). Computer Vision E Image Understanding 2008, pp. 110(3): p. 346-359.

21. Li, D.C.; Su, F. An adaptive symmetry detection algorithm based on local features; 2015.

22. Marola, G. Using symmetry for detecting and locating objects in a picture. Computer Vision Graphics $\mathcal{E}$ Image Processing 1989, 46, 179-195.

23. Wagemans, J. Parallel visual processes in symmetry perception: Normality and pathology. Documenta Ophthalmologica, 95, 359-370.

24. Jenkins, C.; Wan, J.; Holden, E.J.; Dentith, M.; Haederle, M. Application of Radial Symmetry for Caldera Detection. Computing: Techniques and Applications, 2008. DICTA '08.Digital Image, 2009. 
25. Kondra, S.; Petrosino, A.; Iodice, S. Multi-Scale Kernel Operators for Reflection and Rotation Symmetry: Further Achievements. Computer Vision and Pattern Recognition Workshops (CVPRW), 2013 IEEE Conference on, 2013.

26. Patraucean, V.; Gioi, R.G.V.; Ovsjanikov, M. Detection of Mirror-Symmetric Image Patches. Proceedings of the 2013 IEEE Conference on Computer Vision and Pattern Recognition Workshops, 2013.

27. Michaelsen, E.; Muench, D.; Arens, M. Recognition of Symmetry Structure by Use of Gestalt Algebra. Conference on Computer Vision and Pattern Recognition Workshops, 2013.

28. Nagar, R.; Raman, S. [IEEE 2017 IEEE International Conference on Computer Vision Workshop (ICCVW) Venice, Italy (2017.10.22-2017.10.29)] 2017 IEEE International Conference on Computer Vision Workshops (ICCVW) - SymmMap: Estimation of the 2-D Reflection Symmetry Map and Its A. pp. 1715-1724.

29. Yip, R.K.K. A Hough transform technique for the detection of reflectional symmetry and skew-symmetry. Pattern Recognition Letters, 21, 117-130.

30. Nagar, R.; Raman, S. Reflection Symmetry Axes Detection Using Multiple Model Fitting. IEEE Signal Processing Letters, 24, 1438-1442.

31. Lowe.; G., D. Distinctive Image Features from Scale-Invariant Keypoints. International Journal of Computer Vision, 60, 91-110.

32. Ke, N.Y.; Sukthankar, R. PCA-SIFT: a more distinctive representation for local image descriptors. Proceedings of the 2004 IEEE Computer Society Conference on Computer Vision and Pattern Recognition, 2004.

33. Cail, F.; Floru, R.; Roufs, J.A.J.; Boschman, M.C.; Marquie, J.C. EYE MOVEMENTS AND TASK DEMANDS ON VDU; 1987.

34. Kootstra, G.; Bergstrom, N.; Kragic, D. Using Symmetry to Select Fixation Points for Segmentation. 2010.

35. Belongie S., Malik, J.; Puzicha, J. Shape matching and object recognition using shape contexts. IEEE Transactions on Pattern Analysis \& Machine Intelligence, 24, 509-522.

36. I. Rauschert, J.; Liu, Y. Symmetry detection competition: A summary of how the competition is carried out. Proc.IEEE CVPR Workshop Symmetry Detection Real World Images 2011, Workshop on Second Symmetry Detection from Real Images Competition, 1-66.

37. Funk, C., e.a. 2017 ICCV Challenge: Detecting Symmetry in the Wild. 2017.

38. Liu, J.; Slota, G.; Gang, Z.; Wu, Z.; Liu, Y. Symmetry Detection from Real World Images Competition 2013: Summary and Results. Proceedings / CVPR, IEEE Computer Society Conference on Computer Vision and Pattern Recognition. IEEE Computer Society Conference on Computer Vision and Pattern Recognition 2013, Workshop on Second Symmetry Detection from Real Images Competition, 1-6.

39. Nagar, R., S.R. Reflection symmetry detection by embedding symmetry in a graph. ICASSP 2019-2019 IEEE International Conference on Acoustics, Speech and Signal Processing (ICASSP). IEEE, 2019. 